\title{
Cyclin T1 stabilizes expression levels of HIV-1 Tat in cells
}

\author{
Kenichi Imai ${ }^{1}$, Kaori Asamitsu ${ }^{1}$, Ann Florence B. Victoriano ${ }^{1}$, Marni E. Cueno ${ }^{1}$, Koh Fujinaga ${ }^{2}$ and \\ Takashi Okamoto ${ }^{1}$ \\ 1 Department of Molecular and Cellular Biology, Nagoya City University Graduate School of Medical Sciences, Nagoya, Japan \\ 2 Department of Biological Chemistry, University of Michigan Medical School, Ann Arbor, MI, USA
}

\author{
Keywords \\ cyclin T1; HIV; protein stability; Tat; \\ transcription

\section{Correspondence} \\ T. Okamoto, Department of Molecular and \\ Cellular Biology, Nagoya City University \\ Graduate School of Medical Sciences, 1 \\ Kawasumi, Mizuho-cho, Mizuho-ku, Nagoya, \\ Aichi 467-8601, Japan \\ Fax: +81528591235 \\ Tel: +81528538204 \\ E-mail: tokamoto@med.nagoya-cu.ac.jp \\ (Received 2 July 2009, revised 16 \\ September 2009, accepted 5 October \\ 2009) \\ doi:10.1111/j.1742-4658.2009.07424.x
}

Transcription from HIV-1 proviral DNA is a rate-determining step for HIV-1 replication. Interaction between the cyclin T1 (CycT1) subunit of positive transcription elongation factor $\mathrm{b}(\mathrm{P}-\mathrm{TEFb})$ and the Tat transactivator protein of HIV-1 is crucial for viral transcription. CycT1 also interacts directly with the transactivation-responsive element (TAR) located on the $5^{\prime}$ end of viral mRNA, as well as with Tat through the Tat-TAR recognition motif (TRM). These molecular interactions represent a critical step for stimulation of HIV transcription. Thus, Tat and CycT1 are considered to be feasible targets for the development of novel anti-HIV therapies. In this study, we demonstrate that CycT1 is positively involved in the Tat protein stability. Selective degradation of CycT1 by small interfering RNA (siRNA) culminated in proteasome-mediated degradation of Tat and eventual inhibition of HIV-1 gene expression. We noted that the siRNAmediated knockdown of CycT1 could inhibit HIV-1 transcription without affecting cell viability and Tat mRNA levels. These findings clearly indicate that CycT1 is a feasible therapeutic target, and inactivation or depletion of CycT1 should effectively inhibit HIV replication by destabilizing Tat and suppressing Tat-mediated HIV transcription.

\section{Introduction}

HIV-1 is a cytopathic retrovirus that is the primary etiological agent of AIDS and related disorders. HIV-1 transcription is directed by a promoter located in the $5^{\prime}$ long terminal repeat (LTR) of the integrated provirus, and is controlled by cellular factors that bind to the multiple cis-regulatory elements located in the LTR as well as by the virally encoded Tat protein [1]. In the absence of Tat, transcription of HIV mRNAs can be initiated but these cannot be efficiently elongated to produce full-length viral RNA genome [2], making Tat a feasible therapeutic target.

Tat interacts specifically with cyclin $\mathrm{T} 1$ (CycT1), a regulatory partner of cyclin-dependent kinase 9 (CDK9), in the positive transcription elongation factor (P-TEFb) complex, and, together with CycT1, binds cooperatively to the transactivation-responsive element (TAR), in which a bulged RNA loop structure located at the $5^{\prime}$ end of the nascent viral RNA transcripts is used to recruit P-TEFb and promote the transcriptional elongation of HIV [3,4]. Assembly of the TatTAR-P-TEFb complex at the HIV promoter activates the CDK9 kinase activity, which further autophosphorylates $\mathrm{P}-\mathrm{TEFb}$ and hyperphosphorylates the C-terminal domain of RNA polymerase II [5,6], leading to formation of processive elongation complexes that synthesize full-length HIV viral mRNA. Interestingly, murine CycT1, which is very similar to human CycT1 at the amino acid level, could not support HIV-1 transcription [3,7], indicating specificity of the Tat-CycT1 interaction. Moreover, although the nascent P-TEFb

\section{Abbreviations}

CDK9, cyclin-dependent kinase 9; CycT1, cyclin T1; LTR, long terminal repeat; ponA, ponasteron A; P-TEFb, positive transcription elongation factor b; siRNA, small interfering RNA; TAR, transactivation responsive element; TRM, Tat-TAR recognition motif. 
complex includes either CycT2a, CycT2b, CycK $[5,6]$ or CycT1 and CDK9 subunits, only the CycT1/CDK9 heterodimer acts as an HIV-1 Tat-specific transcription factor $[7,8]$.

The development of combination therapy using multiple anti-retroviral drugs for the treatment of HIV-1 infection has led to dramatic therapeutic improvement of anti-HIV therapy and saved the lives of many HIVinfected individuals. However, current anti-HIV therapy does not provide an ultimate cure for patients and may result in drug-resistant viral variants. Therefore, new antiviral therapeutic or preventive approaches are urgently required. Specifically, the transactivation mechanism is an attractive target for the development of new anti-retroviral drug therapies as Tat is required for viral gene expression not only during exponential growth of the virus, but more critically, during activation of the integrated proviral genomes that give rise to drug-resistant strains of HIV-1.

Several studies have demonstrated that Tat-mediated transactivation can be efficiently blocked by various approaches with Tat as a molecular target, including use of anti-Tat intrabodies [9], ribozyme-based inactivation of tat mRNA [10], TAR decoys [11], anti-tat RNA interference [12,13], and others [14-17]. However, a major limitation of these approaches is the sequence variation of the tat gene among virus isolates. Previous reports have shown that RNA interference can be used to successfully downregulate the expression of a number of HIV-1 genes, including gag, pol, vif, nef and tat $[12,13,18-20]$. These results suggest that targeting viral factors required for the HIV-1 lifecycle using small interfering RNA (siRNA) is a viable method for treating HIV-1 infections. However, the high mutation rate of HIV leads to viral mutants that can escape the inhibitory effects of anti-viral siRNAs [21-23]. Thus, a cellular factor that is essential for viral replication, such as CycT1, is a feasible target for antiviral therapy. As CycT1 is fundamental for HIV-1 gene expression, it is an effective target for anti-HIV drug therapy [24-30]. In this report, we demonstrate for the first time that downregulation of CycT1 by siRNA-mediated knockdown also downregulated the steady-state level of Tat protein, leading to suppression of HIV-1 transcription.

\section{Results}

\section{Downregulation of Tat upon cyclin T1 protein depletion}

To examine the effects of CycT1 on HIV-1 gene expression, we used RNA interference against CycT1 to decrease the protein level of CycT1. RNAi against mutant CyT1 (mutCycT1) or GFP was used as control. As shown in Fig. 1A, gene expression from the HIV-1 LTR was upregulated by Tat in a cell line in which Tat expression was under the control of ponasteron $\mathrm{A}$ (ponA) (Fig. 1A). In this cell line, Tat is stably transfected into 293 cell line, and Tat protein expression is detected after $12 \mathrm{~h}$ of ponA treatment [31]. As expected, viral gene expression was significantly downregulated by depleting CycT1 using siRNA. The effects of the specific siRNA and its controls (unrelated siRNA against GFP and mutant siRNA containing two nucleotide mismatches against CycT1) were also examined. As shown in Fig. 1B, CycT1 knockdown dramatically decreased the CycT1 protein level. However, to our surprise, the protein level of Tat was simultaneously downregulated by CycT1 knockdown. In contrast, when control siRNAs were transfected, the Tat protein levels were not changed. Figure 1C shows the dose-dependent depletion of Tat, as well as CycT1, by siRNA against CycT1. No such effect was observed with control siRNAs, GFP or mutCycT1. In Fig. 1D, the Jurkat $\mathrm{T}$ cell line constitutively expressing Tat [32] was similarly examined for the effects of CycT1 knockdown. When CycT1 was knocked down using the CycT1 siRNA, the Tat protein level was also downregulated. In addition, as previously reported by Chiu et al. [28], the protein level of CDK9 was not substantially decreased by CycT1 knockdown (data not shown). No effect was observed with the control siRNA. In addition, the level of a control cellular protein, $\alpha$-tubulin, was not affected by the various siRNAs (Fig. 1B-D).

\section{Restoration of Tat protein level and Tat-mediated HIV-1 transactivation by overexpressing siRNA-resistant CycT1}

We then examined the effect of siRNA against CycT1 by overexpressing exogenous CycT1 ('siRNA-resistant CycT1') in which the transduced CycT1 sequence had been mutated to be resistant to the siRNA. This mutant CycT1 contains five nucleotide substitutions that render the mRNA resistant to siRNA-mediated degradation but do not change the amino acid sequence. Figure 2A shows that, although siRNA against CycT1 could downregulate the protein levels of endogenous CycT1 and thus of Tat (lanes 1 and 2), the levels of Tat, as well as of CycT1, were restored when the siRNA-resistant CycT1 was transduced (lane 3). No effect was found with GFP vector (negative control). We also transfected the siRNA-resistant CycT1 or GFP vector together with HIV-1LTR-luc (Fig. 2B,), and performed a luciferase assay in the presence or absence of siRNA against 


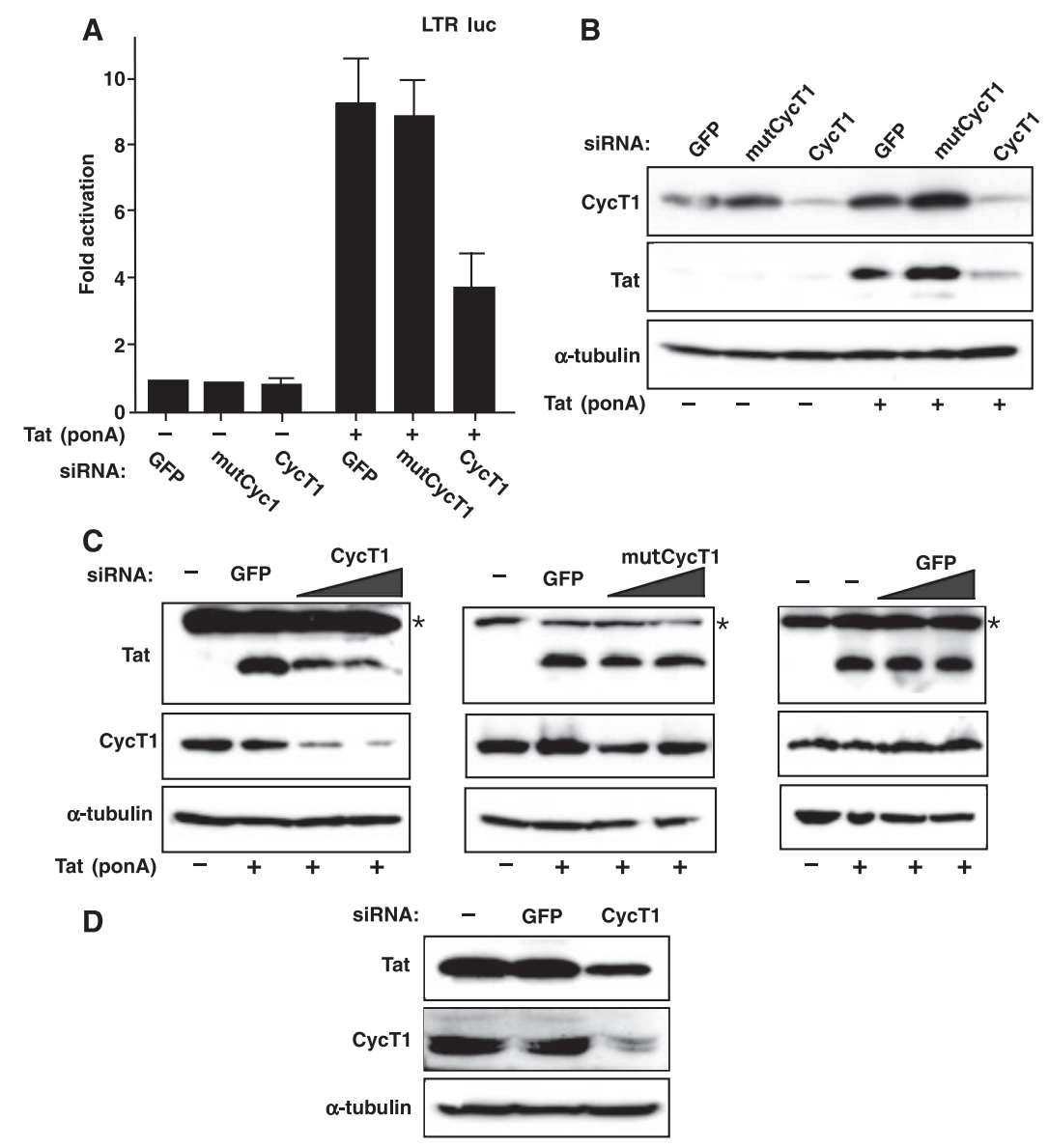

Fig. 1. Inhibition of Tat-mediated HIV-1 transactivation by cyclin T1 protein depletion. 293/Tat cells were transfected with $100 \mathrm{nM}$ siRNAs directed against various portions of CycT1 or GFP (control) mRNAs, together with the HIV-1LTR-luc reporter construct expressing the luciferase gene under the control of the HIV-1 LTR, incubated for $30 \mathrm{~h}$, and treated with ponA (10 $\mu \mathrm{M})$ for an additional $24 \mathrm{~h}$ to induce Tat expression. (A) Luciferase assay. Cells were harvested and the luciferase activity was measured. The data are means and SD for triplicate experiments. (B) Immunoblotting of CycT1 and Tat. CycT1 and Tat protein levels were assessed by immunoblotting using specific antibodies. The blot was stripped and re-probed using anti- $\alpha$-tubulin serum. (C) Downregulation of Tat by CycT1 knockdown. 293/Tat cells were transfected with siRNAs (20-100 nm) targeting CycT1 (left panel), mutCycT1 (mismatched siRNA for CycT1) (center panel) or GFP (right panel). After $30 \mathrm{~h}$ of transfection, cells were either left untreated or treated with ponA $(10 \mu \mathrm{M})$ and incubated for an additional $24 \mathrm{~h}$. Cells were harvested, and both the CycT1 and Tat protein levels were assessed as described in (A). Asterisks indicate non-specific bands. (D) Effect of CycT1 knockdown on the level of Tat protein in Jurkat T cells. Jurkat/Tat cells were transfected with siRNAs (100 nm). After $48 \mathrm{~h}$ of transfection, cells were harvested, and the CycT1 and Tat protein levels were assessed as described in (A).

CycT1. As expected, the siRNA-resistant CycT1, but not the control GFP vector, rescued the Tat-mediated HIV-1 transactivation, consistent with the restoration of Tat protein levels (compare the 2 nd and 3rd columns in Fig. 2B). These results suggest that CycT1 is involved in the protein stabilization of Tat as well as its transcriptional activity.

\section{Effect of CycT1 knockdown on the Tat mRNA level}

We further examined the effect of anti-CycT1 siRNA on the Tat mRNA level. As shown in Fig. 3A, although anti-CycT1 siRNA dramatically reduced the protein level of CycT1, the Tat mRNA level was not significantly downregulated. In addition, non-specific effects of transducing siRNA molecules on the Tat mRNA, as well as its protein levels, were negligible (compare lane 1 with other lanes, and data not shown). Similar results were observed by quantitative RT-PCR for Tat mRNA (Fig. 3B). No effect of siRNA against mutCycT1 or GFP was observed. In addition, the overall effect of siRNA against CycT1 was assessed by measuring the cell growth rate. Figure $3 \mathrm{C}$ shows that there was no significant difference in the relative cell growth rate among cells treated with control siRNA (GFP), mutant siRNA (mutCycT1) or siRNA against CycT1 (CycT1), as 
A

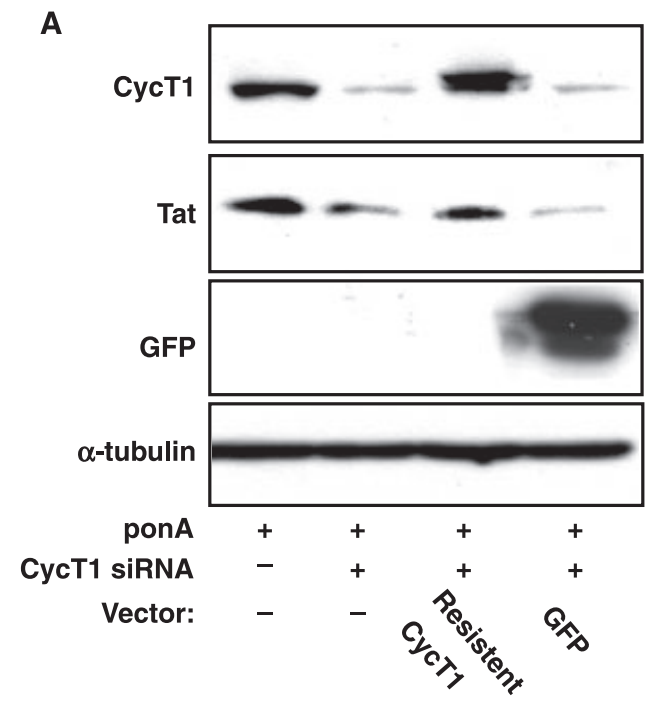

B

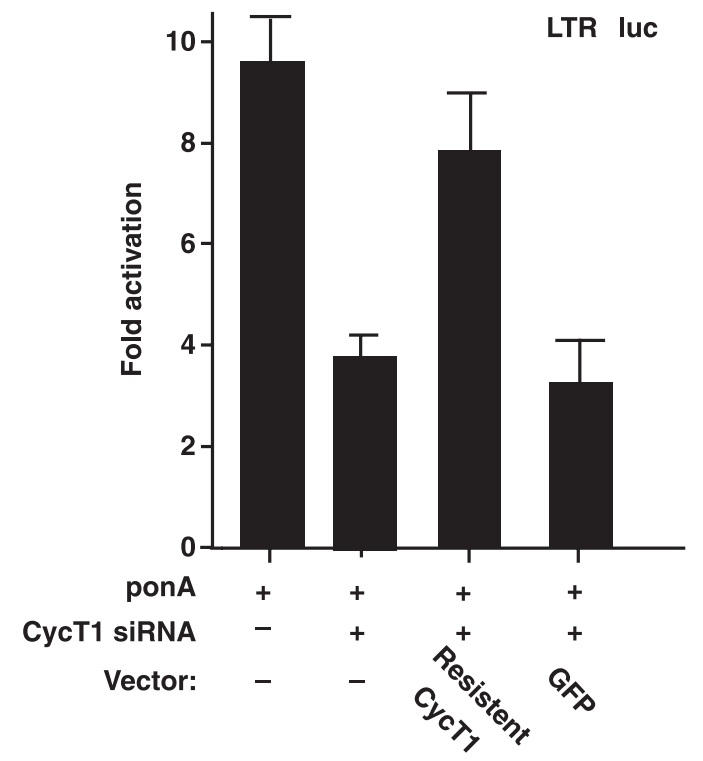

Fig. 2. Restoration of Tat protein and HIV-1 gene expression by siRNA-resistant CycT1. 293/Tat cells were transfected with HIV1LTR-luc together with CycT1 siRNA (100 nM) with or without co-transfection of siRNA-resistant CycT1 or GFP expression vector. After $30 \mathrm{~h}$ of transfection, cells were treated with ponA $(10 \mu \mathrm{M})$ and incubated for additional $24 \mathrm{~h}$. CycT1, Tat and GFP protein levels were assessed by immunoblotting using specific antibodies (A). Cells were harvested and the luciferase activity was measured (B). Values are means and SD.

evaluated by a cell growth assay utilizing WST-1 dye. These findings collectively indicate that downregulation of the Tat protein level by knockdown of CycT1 might be exerted at the level of protein stabilization. Tat protein stability was also examined by a pulse-chase experiment in which CycT1 protein was knocked down by transfection with anti-CycT1 siRNA (Fig. 3D).
293/Tat cells were treated with siRNA and cultured in the presence of ponA to induce Tat expression. The cultured cells were then incubated with medium that was free of methionine and cysteine, which was subsequently replaced with medium containing ${ }^{35}$ S-labeled methionine and cysteine ('pulse'), followed by cultivation in the presence of excess amounts of methionine and cysteine ('chase'). Cell lysates were prepared from these cell cultures and subjected to immunoprecipitation using the epitope tag (Flag) present in the Tat protein.

As shown in Fig. 3D, the protein level of Tat was dramatically decreased by the siRNA against CycT1 (left panel), but no significant change in the Tat protein level was detected in cells treated with the control siRNA (right panel). Altogether, these results indicated that CycT1 protein knockdown affects the Tat protein stability.

\section{Involvement of proteasome in Tat protein degradation}

The above observations led us to examine whether the level of Tat protein is determined by proteolytic degradation. Figure 4A shows the extent of downregulation of Tat protein in the presence of anti-CycT1 siRNA in the presence of various protease inhibitors. Of these inhibitors, the proteasome inhibitors including MG132 and lactacystin increased the steady-state level of Tat protein, but no such effect was seen with other protease inhibitors such as leupeptin, pepstatin $\mathrm{A}$ and aprotinin. However, the lysosomal inhibitor $\mathrm{NH}_{4} \mathrm{Cl}$ showed a moderate effect. These observations indicate that the proteasome pathway might be primarily involved in degradation of Tat protein, and are concomitant with the siRNA-mediated downregulation of CycT1. However, the siRNA-mediated CycT1 degradation was not restored by proteasome inhibitors, presumably because the CycT1 degradation takes place at the level of its mRNA by the action of siRNA. No such effects of proteasome inhibitors were observed in the absence of siRNA against CycT1 (Fig. 4B).

\section{Discussion}

Tat plays an essential role in HIV gene expression and viral replication [1], and stimulates transcription from HIV provirus by recruiting $\mathrm{P}-\mathrm{TEFb}$ through direct binding with CycT1, a regulatory subunit of $\mathrm{P}-\mathrm{TEFb}$ [2-4]. As transcription is a rate-determining step of viral replication, and Tat is unique in that it specifically stimulates transcription of viral gene expression, Tat has been considered to be an appropriate target for novel anti-HIV therapy. However, none of the anti-Tat 
A

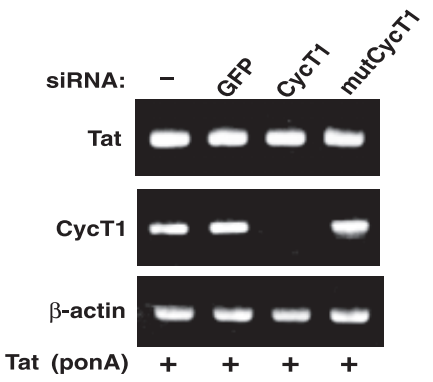

C

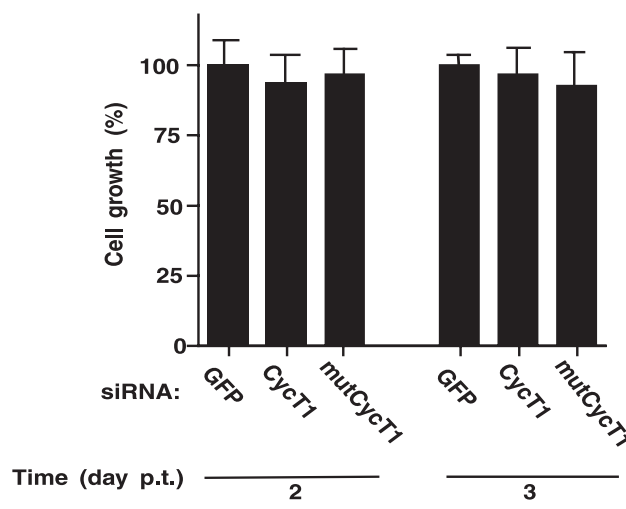

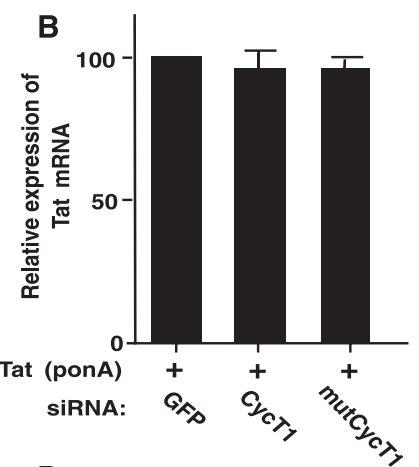

D

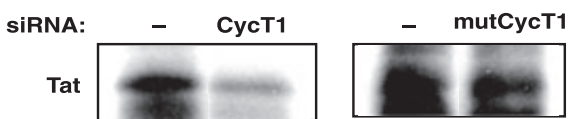

Fig. 3. Effects of CycT1 knockdown on Tat mRNA levels. (A,B) Effects of CycT1 knockdown on Tat mRNA. 293/Tat cells were transfected with siRNAs $(100 \mathrm{nM})$ for $30 \mathrm{~h}$, and treated with ponA $(10 \mu \mathrm{M})$ to induce Tat expression. After $24 \mathrm{~h}$ of ponA stimulation, total RNA was purified from the cell culture, and subjected to RT-PCR using gene-specific primers (A) or real-time RT-PCR analysis using Tat primer/probe mixtures (B). (C) Effects of CycT1 knockdown on cell growth. 293/Tat cells were transfected with siRNAs (100 nm) for 48 or $72 \mathrm{~h}$. Cell viability was determined by the WST-1 method. No significant effects on cell growth and viability were noted. (D) Tat protein stability depends on the presence of CycT1. 293/Tat cells were treated with siRNAs against CycT1 or mutCycT1 (100 nM each) for $30 \mathrm{~h}$, cultured in the presence of ponA for $8 \mathrm{~h}$, replaced in a methionine- and cysteine-free culture medium and further cultured for 30 min. The cultured cells were then incubated in the presence of ${ }^{35}$ S-labeled methionine and cysteine for an additional $4 \mathrm{~h}$ ('pulse'), followed by cultivation in the presence of excess amounts ( $2 \mathrm{~mm}$ ) of cold methionine and cysteine for $14 \mathrm{~h}$ ('chase'). The cells were lysed, and Tat was immunoprecipitated using anti-Flag (M2) affinity gel beads and visualized by fluorography to detect radioactivity as described in Experimental procedures. The experiments were repeated, and representative data are shown.

strategies developed to date, including low-molecularmass Tat antagonists [15], dominant-negative Tat $[14,33]$, anti-Tat single chain intrabodies [9], inhibitory Tat peptides [16,17,34,35], TAR decoys [11], anti-tat hammerhead ribozymes [10] or siRNAs against Tat $[12,13,19]$, have been found to be clinically applicable, presumably due to lack of specificity or the fact that Tat action has to be completely abolished in order to block its transcriptional activity. Similarly, as Tat is a viral protein and is subject to mutational changes in accordance with the presence of its inhibitor, new strategies may involve considering host factors such as CycT1 as molecular targets. Tat is a natively unfolded or naturally disordered protein, and is rapidly degraded when expressed alone [36]. A recent report on a tripartite complex formation among CycT1, equine infectious anemia virus Tat and TAR provided structural insights on the mutual stabilization of the molecules [37]. Interestingly, the CycT1 protein level is increased when cells are infected by HIV-1 and when Tat is produced $[38,39]$, suggesting that the protein stabilities of Tat and CycT1 depend on each other. We therefore examined whether the Tat protein level was downregulated when CycT1 is downregulated. In this study, we found that, when CycT1 was knocked down, Tat protein was dramatically destabilized and subsequently degraded by the proteasome pathway.

Several approaches have been used to block HIV transcription by targeting CycT1, including the use of small compounds or mutant proteins that disrupt the interaction between Tat, TAR and CycT1 $[24,30]$. In addition, several CycT1-binding proteins and their truncated mutants have been examined as potential inhibitors of Tat transactivation [25,27]. Moreover, Bai 
Fig. 4. Effects of protease inhibitors on Tat protein degradation. 293/Tat cells were transfected with CycT1 siRNA (100 nM) for $30 \mathrm{~h}$, and protease inhibitors was added to the culture together with ponA $(10 \mu \mathrm{m})$ and further cultured for $24 \mathrm{~h}$. The cells were harvested and both Tat and CycT1 protein levels were analyzed by immunoblotting. The inhibitors used were MG132 (5 $\mu \mathrm{M})$, lactacystin $(5 \mu \mathrm{M})$, leupeptin $\left(500 \mu \mathrm{g} \cdot \mathrm{mL}^{-1}\right)$, pepstatin $\mathrm{A}(20 \mu \mathrm{M})$, aprotinin $(10 \mu \mathrm{M})$ and $\mathrm{NH} 4 \mathrm{Cl}(5 \mathrm{~mm})$. Immunoblotting was performed on cell cultures with (A) or without (B) siRNA against CycT1.

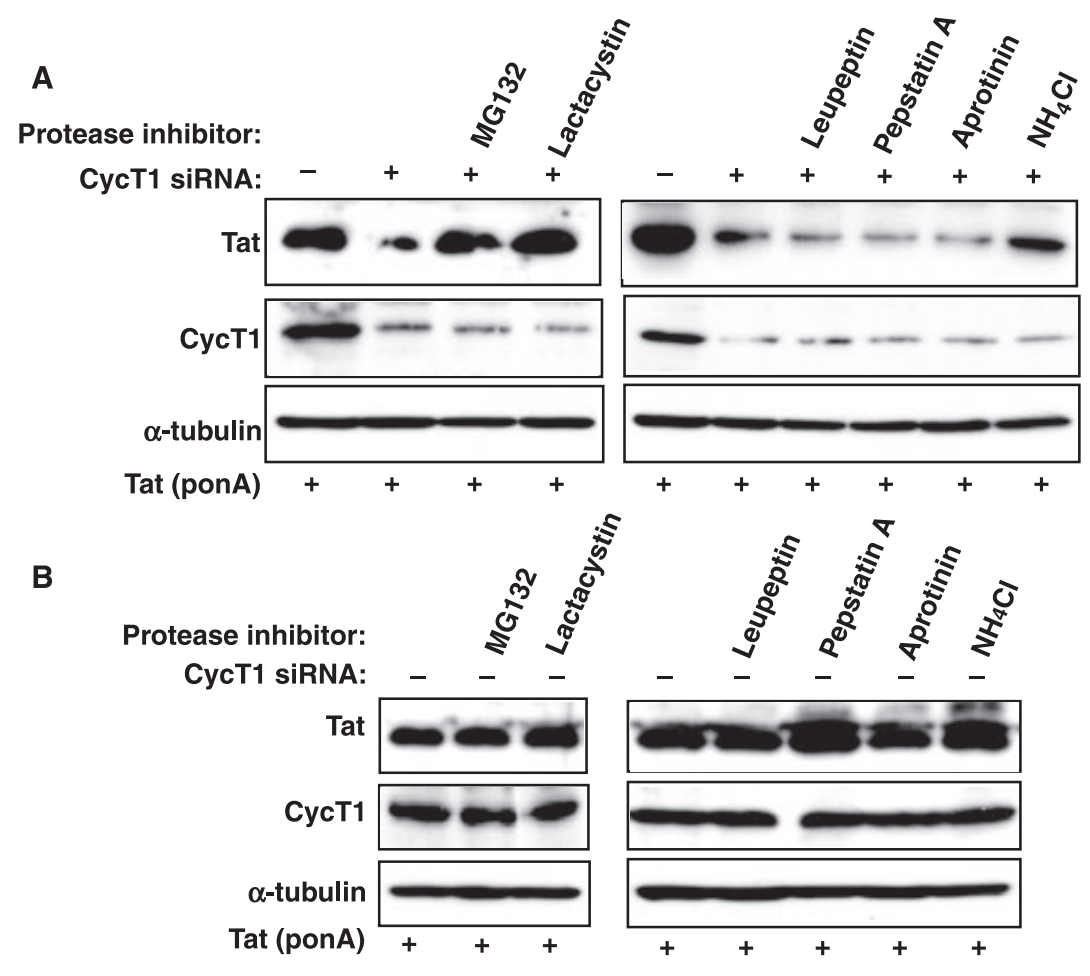

et al. [26] demonstrated that transduction of antiCycT1 intrabodies could render cells resistant to HIV-1 replication by effectively inhibiting Tat-mediated HIV-1 transactivation. Furthermore, two independent studies $[28,29]$ reported that siRNA directed to CycT1 successfully downregulated Tat action and HIV-1 viral replication, although they did not demonstrate accelerated Tat protein degradation. These results support an essential role for CycT1 in Tat transactivation and HIV-1 replication.

It was anticipated that downregulation of CycT1 would cause non-specific effects on cell viability or function given the involvement of P-TEFb in the transcription of many cellular genes [2]. However, other studies [28,29] demonstrated that knockdown of CycT1 led to inhibition of HIV-1 replication but did not affect $\mathrm{P}-\mathrm{TEFb}$ kinase activity and cell viability, as observed in this study (Fig. 3C). This indicates that a very low level of P-TEFb kinase activity might be sufficient for the maintenance of cell viability, or the cells could compensate for lower P-TEFb protein levels by converting kinase-inactive $\mathrm{P}-\mathrm{TEFb}$ into active forms [28]. It has also been reported that, although CycT1 knockdown in the human monocytic cell line MM6 by small hairpin RNA inhibited Tat activation, MM6 cell growth was not affected by the depletion of CycT1 [40]. However, as induction of substantial numbers of genes associated with phorbol 12-myristate 13-acetate-mediated stimula- tion of MM6 and CD4 ${ }^{+} \mathrm{T}$ cells is known to require CycT1 [40,41], downregulation of CycT1 may have some side effects in such cell lineages. It is possible that other CycT family proteins such as CycT2a and CycT2b could functionally substitute for CycT1. Moreover, as hexamethylene bis-acetamide inducible protein 1 is known to bind CycT1 at a similar location as Tat [42], it is possible that hexamethylene bis-acetamide inducible protein 1 protein has been degraded upon CycT1 knockdown as transcriptional activity was rescued by the siRNA-resistant CycT1, as demonstrated in this study (Fig. 2).

We also found that downregulation of Tat protein could be blocked by certain protease inhibitors, namely MG132 and lactacystin, which are both proteasome inhibitors, and to a lesser extent by $\mathrm{NH} 4 \mathrm{Cl}$, a lysosome inhibitor. It has been reported that the E3 ubiquitin ligase $\mathrm{Hdm} 2$ binds to Tat, mediates its polyubiquitylation, and supports HIV-1 transactivation by Tat [43]. Although we attempted to specify the posttranslational modification of Tat that is responsible for proteolytic cleavage, we did not obtain reproducible results, even using proteasome inhibitors. Interestingly, a recent report by Jadlowsky et al. [30] demonstrated that a dominant-negative CycT1 mutant that binds to Tat but cannot support Tat-mediated HIV-1 transactivation leads to degradation of Tat in addition to its own degradation when transduced into cells. These 
results, together with our findings (Fig. 4), suggest that is it reasonable to assume that any abortive protein complex involving Tat and CycT1, but lacking functional activity, is degraded by the proteasome pathway. However, the distinct roles of various Tat modifications such as poly- and monoubiquitination required clarification, and the ubiquitination enzyme(s) need to be identified. Nevertheless, our findings clearly demonstrate that downregulation of Tat-mediated HIV-1 transactivation could primarily be due to the downregulation of CycT1, although the possibility of upregulation of Tat action by polyubiquitination remains to be explored. Thus, reagents that inhibit the interaction between Tat and CycT1 could work as feasible Tat inhibitors.

\section{Experimental procedures}

\section{Cell culture}

The establishment of a ponA-inducible cell line expressing Tat has been described previously [31]. In this cell line, Tat was stably transfected into 293 cells, and the expression of Tat protein is under the stringent control of ponasteron $\mathrm{A}$ (Invitrogen, Carlsbad, CA, USA), a homolog of the insect hormone 20-OH-ecdysone. The cells were maintained at $37^{\circ} \mathrm{C}$ in Dulbecco's modified Eagle's medium (Sigma, St Louis, MO, USA) with $10 \%$ fetal bovine serum (Sigma), $500 \mu \mathrm{g} \cdot \mathrm{mL}^{-1} \mathrm{G} 418$ (Invitrogen) and $450 \mu \mathrm{g} \cdot \mathrm{mL}^{-1}$ zeocine. The Jurkat/Tat $\mathrm{T}$ cells, which constitutively express Tat protein [32], were maintained in RPMI-1640 (Sigma) with $10 \%$ fetal bovine serum and $\mathrm{G} 418\left(800 \mu \mathrm{g} \cdot \mathrm{mL}^{-1}\right)$.

\section{RNA interference and transfection}

siRNAs with two thymidine residues (dTdT) at the $3^{\prime}$ end were chemically synthesized (Takara Bio, Ohtsu, Japan). The target sequences were as follows: CycT1, 5'-UC CCUUCCUGAUACUAGAA-3' (nucleotide positions 347 -365); mutant CycT1, 5'-UCCCUUCCGUAUACUAGA A-3' [28]; GFP, 5'-GGCUACGUCCAGGAGCGCACC-3'. Underlined residues indicated the sequence that is mismatched with respect to CycT1. Transfection of siRNA into 293/Tat cells was performed using Lipofectamine 2000 (Invitrogen) as described previously [31]. Jurkat/Tat cells were transfected using Nucleofector ${ }^{\mathrm{TM}}$ kit $\mathrm{V}$ for Jurkat cells (Amaxa Biosystems, Walkersville, MD, USA) as described previously [44]. Briefly, $3 \times 10^{6}$ cells were mixed with $100 \mathrm{nM}$ of siRNA in $100 \mu \mathrm{L}$ of Nucleofector ${ }^{\mathrm{TM}}$ solution V. These samples were transferred into a transfection cuvette and subjected to electroporation using program T-14. After electroporation, cells were immediately transferred to $2.0 \mathrm{~mL}$ of culture medium, and cultured in six-well plates at $37^{\circ} \mathrm{C}$ for $48 \mathrm{~h}$.

\section{Plasmid construction}

The HIV-1 LTR-based luciferase expression plasmid HIV1LTR-luc (containing the entire U3 and R sequences of HIV-1) has been described previously [44,45]. The mutated CycT1 expression vector, containing five nucleotide substitutions, including T361G, A362C, A364C, A365T and $\mathrm{G} 366 \mathrm{C}$, that do not change the corresponding amino acid but render CycT1 resistant to the siRNA, was constructed using a site-directed mutagenesis kit (QuickChange, Stratagene, La Jolla, CA, USA) with pcDNA-CycT1 [31] as a template and with the following mutagenesis oligonucleotide primer pair: forward, 5'-CCCTTCCTGATACGCG CTCTGAGGCTTATTTG-3'; reverse, 5'-CAAATAAGC CTCAGAGCGCGTATCAGGAAGGG-3'. All constructs were confirmed by dideoxynucleotide sequencing using an ABI PRISM ${ }^{\mathrm{TM}}$ Dye Terminator Cycle Sequencing Ready Kit (Perkin-Elmer, Waltham, MA, USA) on an Applied Biosystems 313 automated DNA sequencer (Applied Biosystems, Foster City, CA, USA).

\section{Immunoblot assays}

Immunoblotting was performed as described previously [40]. Briefly, cells were harvested using lysis buffer (25 mM Hepes/ $\mathrm{NaOH}$ pH 7.9, $150 \mathrm{~mm} \mathrm{NaCl}, 1.5 \mathrm{~mm} \mathrm{MgCl}_{2}, 0.2 \mathrm{~mm}$ EDTA, $0.3 \%$ Nonidet P-40, $1.0 \mathrm{~mm}$ dithiothreitol, $0.5 \mathrm{~mm}$ phenylmethylsulfonyl fluoride). After centrifugation at $4{ }^{\circ} \mathrm{C}$ for $20 \mathrm{~min}$ at $15000 \mathrm{~g}$, the supernatant proteins were separated by SDS-PAGE and transferred to nitrocellulose membrane (Hybond-C, Amersham, Piscataway, NJ, USA). The membrane was probed using antibodies against Flag (Sigma), Tat (ZeptoMetrix Co., Buffalo, NY, USA) or CycT1 (Santa Cruz Biotechnology, Santa Cruz, CA, USA), and immunoreactive proteins were visualized by enhanced chemiluminescence (SuperSignal, Pierce, Rockford, IL, USA).

\section{Luciferase assay}

The luciferase assay using HIV-1LTR-luc was performed as described previously [46]. The transfected cells were harvested and the extracts were subjected to luciferase assay using the Luciferase Assay System ${ }^{\mathrm{TM}}$ (Promega, Madison, WI, USA). All experiments were performed in triplicate, and the data are presented as the fold increase in luciferase activity (means $\pm \mathrm{SD}$ ) relative to the control for three independent transfections.

\section{Preparation of mRNA and RT-PCR}

Total cellular RNA was prepared from each cell culture using RNeasy (Qiagen, Valencia, CA, USA). For cDNA synthesis, $1.0 \mu \mathrm{g}$ of total RNA was reverse-transcribed using an oligo(dT) primer and Superscript II reverse transcriptase 
(Invitrogen). The cDNA was then amplified from each RNA sample using Taq master mix (Qiagen) and gene-specific primers. The primer sequences for each amplified gene were: Tat forward, 5'-GCATCTCCTATGGCAGGAAG-3'; Tat reverse, 5'-GGACCGGATCTGTCTCTGTC-3'; CycT1 forward, 5'-GTCCCTCATTCGAAACTGGA-3'; CycT1 reverse, $\quad 5^{\prime}$-GTTCTGATGGCAGAGGTGGT-3'; $\beta$-actin forward, 5'-CAGCAAGCAGGAGTATGACGA-3'; $\beta$-actin reverse, 5'-GTGGACTTGGGAGAGGACTGG-3'. The number of cycles was chosen to enable linear amplification of the cDNA under study. The PCR products were separated on $1.5 \%$ agarose gels and visualized by ethidium bromide staining.

Quantitative real-time RT-PCR was performed as described previously [31]. The oligonucleotide primers and probe for Tat were synthesized by Applied Biosystems. The primer and probe sequences for Tat were $5^{\prime}$-GGAG GAGGGTTGCTTTGATAGAG-3' (forward) and 5'-AAA GCCTTAGGCATCTCCTATGG-3' (reverse); probe, 5'-C TTCGTCGCTGTCTCCGCTTCTTCC-3'. The oligonucleotide primers and probe for $\beta$-actin genes were purchased from Assays-on-Demand ${ }^{\mathrm{TM}}$ (Applied Biosystems). Quantitative measurements of each mRNA level were performed in triplicate. The expression levels of test genes were normalized to the mRNA level of $\beta$-actin, as an internal control, in the same mRNA sample.

\section{Cell proliferation assay}

The extents of cell proliferation were evaluated using 2-(4iodophenyl)-3-(4-nitrophenyl)-5-(2,4-disufophenyl)-2H-tetrazolium (WST-1) (Roche, Indianapolis, IN, USA) according to the manufacturer's protocol. In brief, 293/Tat cells were transfected with siRNAs in a 96-well plate for 48 or $72 \mathrm{~h}$, incubated for a further $4 \mathrm{~h}$ in the presence of WST-1, and the level of water-soluble formazan produced was measured at $450 \mathrm{~nm}$ by spectrophotometry.

\section{Labeling of cellular protein with ${ }^{35} \mathrm{~S}$-labeled amino acid and immunoprecipitation of Tat}

After $30 \mathrm{~h}$ of cultivation, the cells were transfected with siRNA against CycT1 or mutCycT1 (100 nM each), and treated with ponA for $8 \mathrm{~h}$. The culture was then maintained in methionine/cysteine-free medium for $30 \mathrm{~min}$ and pulselabeled with $200 \mu \mathrm{Ci} \cdot \mathrm{mL}^{-1}$ of $\left[{ }^{35} \mathrm{~S}\right]$ in vitro Cell Labeling Mix (GE Healthcare, Piscataway, NJ, USA) containing ${ }^{35} \mathrm{~S}$-labeled cysteine and methionine for $4 \mathrm{~h}$. The cells were washed quickly twice using $\mathrm{NaCl} / \mathrm{P}_{\mathrm{i}}$, and incubated for an additional $14 \mathrm{~h}$ in Dulbecco's modified Eagle's medium supplemented with excess methionine and cysteine ( $2 \mathrm{mM}$ each). Cells were harvested using lysis buffer ( $25 \mathrm{~mm}$ Hepes/ $\mathrm{NaOH}$ $\mathrm{pH}$ 7.9, $150 \mathrm{~mm} \mathrm{NaCl}, 1.5 \mathrm{~mm} \mathrm{MgCl}_{2}, 0.2 \mathrm{~mm}$ EDTA, $0.3 \%$ NP-40, $1 \mathrm{~mm}$ dithiothreitol, $0.5 \mathrm{~mm}$ phenylmethylsulfonyl fluoride) and incubated at $4{ }^{\circ} \mathrm{C}$ for $20 \mathrm{~min}$ with gentle rotation. The cell lysates were centrifuged at $15000 \mathrm{~g}$ for $20 \mathrm{~min}$. The supernatant was incubated with anti-Flag M2 affinity gel beads (Sigma) for $2 \mathrm{~h}$ at $4{ }^{\circ} \mathrm{C}$. Immune complexes were washed three times with $1.0 \mathrm{~mL}$ of lysis buffer, and antibody-bound proteins were dissolved by boiling in $2 \times$ Laemmli sample buffer. After centrifugation at $4{ }^{\circ} \mathrm{C}$ for $5 \mathrm{~min}$ at $15000 \mathrm{~g}$, the supernatant proteins were separated by SDS-PAGE and visualized by autoradiography.

\section{Acknowledgements}

This work was supported by grants-in-aid from the Ministry of Health, Labor and Welfare of Japan, the Ministry of Education, Culture, Sports, Science and Technology of Japan, and the Japan Human Sciences Foundation.

\section{References}

1 Jones KA (1997) Taking a new TAK on Tat transactivation. Genes Dev 11, 2593-2599.

2 Price DH (2000) P-TEFb, a cyclin-dependent kinase controlling elongation by RNA polymerase II. Mol Cell Biol 20, 2629-2634.

3 Garber ME, Wei P, KewalRamani VN, Mayall TP, Herrmann CH, Rice AP, Littman DR \& Jones KA (1998) The interaction between HIV-1 Tat and human cyclin $\mathrm{T} 1$ requires zinc and a critical cysteine residue that is not conserved in the murine CycT1 protein. Genes Dev 12, 3512-3527.

4 Wei P, Garber ME, Fang SM, Fischer WH \& Jones KA (1998) A novel CDK9-associated C-type cyclin interacts directly with HIV-1 Tat and mediates its highaffinity, loop-specific binding to TAR RNA. Cell 92, 451-462.

5 Mancebo HS, Lee G, Flygare J, Tomassini J, Luu P, Zhu Y, Peng J, Blau C, Hazuda D, Price D et al. (1997) P-TEFb kinase is required for HIV Tat transcriptional activation in vivo and in vitro. Genes Dev 11, 2633-2644.

6 Zhu Y, Pe'ery T, Peng J, Ramanathan Y, Marshall N, Marshall T, Amendt B, Mathews MB \& Price DH (1997) Transcription elongation factor P-TEFb is required for HIV-1 tat transactivation in vitro. Genes Dev 11, 2622-2632.

7 Kwak YT, Ivanov D, Guo J, Nee E \& Gaynor RB (1999) Role of the human and murine cyclin $\mathrm{T}$ proteins in regulating HIV-1 tat-activation. J Mol Biol 288, 57-69.

8 Peng J, Zhu Y, Milton JT \& Price DH (1998) Identification of multiple cyclin subunits of human P-TEFb. Genes Dev 12, 755-762.

9 Mhashilkar AM, Bagley J, Chen SY, Szilvay AM, Helland DG \& Marasco MA (1995) Inhibition of HIV-1 Tat-mediated LTR transactivation and HIV-1 
infection by anti-Tat single chain intrabodies. EMBO $\mathrm{J}$ 14, 1542-1551.

10 Jackson WH Jr, Moscoso H, Nechtman JF, Galileo DS, Garver FA \& Lanclos KD (1998) Inhibition of HIV-1 replication by an anti-tat hammerhead ribozyme. Biochem Biophys Res Commun 245, 81-84.

11 Bohjanen PR, Colvin RA, Puttaraju M, Been MD \& Garcia-Blanco MA (1996) A small circular TAR RNA decoy specifically inhibits Tat-activated HIV-1 transcription. Nucleic Acids Res 24, 3733-3738.

12 Coburn GA \& Cullen BR (2002) Potent and specific inhibition of human immunodeficiency virus type 1 replication by RNA interference. J Virol 76, 9225-9231.

13 Surabhi RM \& Gaynor RB (2002) RNA interference directed against viral and cellular targets inhibits human immunodeficiency virus type 1 replication. $J$ Virol 76, 12963-12973.

14 Green M, Ishino M \& Loewenstein PM (1989) Mutational analysis of HIV-1 Tat minimal domain peptides: identification of trans-dominant mutants that suppress HIV-LTR-driven gene expression. Cell 58, 215-223.

15 Hsu MC, Schutt AD, Holly M, Slice LW, Sherman MI, Richman DD, Potash MJ \& Volsky DJ (1991) Inhibition of HIV replication in acute and chronic infections in vitro by a Tat antagonist. Science 254, 1799-1802.

16 Hamy F, Felder ER, Heizmann G, Lazdins J, Aboulela F, Varani G, Karn J \& Klimkait T (1997) An inhibitor of the Tat/TAR RNA interaction that effectively suppresses HIV-1 replication. Proc Natl Acad Sci USA 94, 3548-3553.

17 Okamoto H, Cujec TP, Peterlin BM \& Okamoto T (2000) HIV-1 replication is inhibited by a pseudo-substrate peptide that blocks Tat transactivation. Virol 270, 337-344.

18 Jacque JM, Triques K \& Stevenson M (2002) Modulation of HIV-1 replication by RNA interference. Nature 418, 435-438.

19 Lee MT, Coburn GA, McClure MO \& Cullen BR (2003) Inhibition of human immunodeficiency virus type 1 replication in primary macrophages by using Tat- or CCR5-specific small interfering RNAs expressed from a lentivirus vector. J Virol 77, 11964-11972.

20 Novina CD, Murray MF, Dykxhoorn DM, Beresford PJ, Riess J, Lee SK, Collman RG, Lieberman J, Shankar P \& Sharp PA (2002) siRNA-directed inhibition of HIV-1 infection. Nat Med 8, 681-686.

21 Boden D, Pusch O, Lee F, Tucker L \& Ramratnam B (2003) Human immunodeficiency virus type 1 escape from RNA interference. $J$ Virol 77, 11531-11535.

22 Das AT, Brummelkamp TR, Westerhout EM, Vink M, Madiredjo M, Bernards R \& Berkhout B (2004) Human immunodeficiency virus type 1 escapes from RNA interference-mediated inhibition. $J$ Virol 78, 2601-2605.

23 Westerhout EM, Ooms M, Vink M, Das AT \& Berkhout B (2005) HIV-1 can escape from RNA interference by evolving an alternative structure in its RNA genome. Nucleic Acids Res 33, 796-804.

24 Fujinaga K, Irwin D, Geyer M \& Peterlin BM (2002) Optimized chimeras between kinase-inactive mutant Cdk9 and truncated cyclin T1 proteins efficiently inhibit Tat transactivation and human immunodeficiency virus gene expression. J Virol 76, 10873-10881.

25 Young TM, Wang Q, Pe'ery T \& Mathews MB (2003) The human I-mfa domain-containing protein, HIC, interacts with cyclin T1 and modulates $\mathrm{P}$ -TEFb-dependent transcription. Mol Cell Biol 23, 6373-6384.

26 Bai J, Sui J, Zhu RY, Tallarico AS, Gennari F, Zhang D \& Marasco WA (2003) Inhibition of Tat-mediated transactivation and HIV-1 replication by human antihCyclinT1 intrabodies. J Biol Chem 278, 1433-1442.

27 Hoque M, Tian B, Mathews MB \& Pe'ery T (2005) Granulin and granulin repeats interact with the Tat/ P-TEFb complex and inhibit Tat transactivation. $J$ Biol Chem 280, 13648-13657.

28 Chiu YL, Cao H, Jacque JM, Stevenson M \& Rana TM (2004) Inhibition of human immunodeficiency virus type 1 replication by RNA interference directed against human transcription elongation factor P-TEFb (CDK9/cyclinT1). J Virol 78, 2517-2529.

29 Li Z, Xiong Y, Peng Y, Pan J, Chen Y, Wu X, Hussain S, Tien P \& Guo D (2005) Specific inhibition of HIV-1 replication by short hairpin RNAs targeting human cyclin T1 without inducing apoptosis. FEBS Lett 579, 3100-3106.

30 Jadlowsky JK, Nojima M, Schulte A, Geyer M, Okamoto T \& Fujinaga K (2008) Dominant negative mutant cyclin T1 proteins inhibit HIV transcription by specifically degrading Tat. Retrovirology 5, 63-77.

31 Imai K, Nakata K, Kawai K, Hamano T, Mei N, Kasai H \& Okamoto T (2005) Induction of 8-oxoguanine DNA glycosylase 1 gene expression by HIV-1 Tat. $J$ Biol Chem 280, 26701-26713.

32 Caputo A, Sodroski JG \& Haseltine WA (1990) Constitutive expression of HIV-1 tat protein in human Jurkat $\mathrm{T}$ cells using a $\mathrm{BK}$ virus vector. J Acquir Immune Defic Syndr 3, 372-379.

33 Pearson L, Garcia J, Wu F, Modesti N, Nelson J \& Gaynor R (1990) A transdominant tat mutant that inhibits tat-induced gene expression from the human immunodeficiency virus long terminal repeat. Proc Natl Acad Sci USA 87, 5079-5083.

34 Agbottah E, Zhang N, Dadgar S, Pumfery A, Wade JD, Zeng C \& Kashanchi F (2006) Inhibition of HIV-1 virus replication using small soluble Tat peptides. Virol 345, 373-389.

35 D'Orso I, Grunwell JR, Nakamura RL, Das C \& Frankel AD (2008) Targeting tat inhibitors in the assembly of human immunodeficiency virus type 1 transcription complexes. J Virol 82, 9492-9504. 
36 Shojania S \& O'Neil JD (2006) HIV-1 Tat is a natively unfolded protein: the solution conformation and dynamics of reduced HIV-1 Tat-(1-72) by NMR spectroscopy. J Biol Chem 281, 8347-8356.

37 Anand K, Schulte A, Vogel-Bachmayr K, Scheffzek K \& Geyer M (2008) Structural insights into the cyclin T1-Tat-TAR RNA transcription activation complex from EIAV. Nat Struct Mol Biol 15, 1287-1292.

38 Liou LY, Herrmann CH \& Rice AP (2002) Transient induction of cyclin $\mathrm{T} 1$ during human macrophage differentiation regulates human immunodeficiency virus type 1 Tat transactivation function. J Virol 76, 1057910587.

39 Liou LY, Herrmann CH \& Rice AP (2004) Human immunodeficiency virus type 1 infection induces cyclin T1 expression in macrophages. $J$ Virol 78, 8114-8119.

$40 \mathrm{Yu} \mathrm{W}$, Wang Y, Shaw CA, Qin XF \& Rice AP (2006) Induction of the HIV-1 Tat co-factor cyclin T1 during monocyte differentiation is required for the regulated expression of a large portion of cellular mRNAs. Retrovirology 9, 32-47.

$41 \mathrm{Yu}$ W, Ramakrishnan R, Wang Y, Chiang K, Sung TL \& Rice AP (2008) Cyclin T1-dependent genes in activated $\mathrm{CD} 4{ }^{+} \mathrm{T}$ and macrophage cell lines appear enriched in HIV-1 co-factors. PLOS ONE 3, e3146.

42 Schulte A, Czudnochowski N, Barboric M, Schönichen A, Blazek D, Peterlin BM \& Geyer M (2005) Identification of a cyclin T-binding domain in Hexim 1 and biochemical analysis of its binding competition with HIV-1 Tat. J Biol Chem 280, 24968-24977.

43 Brès V, Kiernan RE, Linares LK, Chable-Bessia C, Plechakova O, Tréand C, Emiliani S, Peloponese JM, Jeang KT, Coux O et al. (2003) A non-proteolytic role for ubiquitin in Tat-mediated transactivation of the HIV-1 promoter. Nat Cell Biol 5, 754-761.

44 Imai K \& Okamoto T (2006) Transcriptional repression of human immunodeficiency virus type 1 by AP-4. $J$ Biol Chem 281, 12495-12505.

45 Okamoto T \& Wong-Staal F (1986) Demonstration of virus-specific transcriptional activators in cells infected with HTLV-III by an in vitro cell-free system. Cell 47, 29-35.

46 Imai K, Ochiai K \& Okamoto T (2009) Reactivation of latent HIV-1 infection by the periodontopathic bacterium Porphyromonas gingivalis involves histone modification. J Immunol 186, 1788-1793. 